\title{
THE ATIYAH-CHERN CHARACTER YIELDS THE SEMIREGULARITY MAP AS WELL AS THE INFINITESIMAL ABEL-JACOBI MAP
}

\author{
RAGNAR-OLAF BUCHWEITZ AND HUBERT FLENNER
}

\section{INTRODUCTION}

The purpose of this work is to construct a general semiregularity map for cycles on a complex analytic or algebraic manifold and to show that such semiregularity map can be obtained from the classical tool of the Atiyah-Chern character. The first part of the paper is fairly detailed, deducing the existence and explicit form of a generalized semiregularity map from known results and constructions. As a corollary we obtain in the second part as well a description of the infinitesimal AbelJacobi map for smooth cycles as the leading term of this generalized semiregularity map, indicate why for locally complete intersections the appropriate component of our semiregularity map coincides with the one constructed by Bloch [Blo], and give applications to embedded deformations and deformations of coherent modules.

At the request of several participants at this conference, we restrict ourselves mainly to the case of a smooth ambient space, avoiding thus the formidable technical machinery of resolvents, powers of cotangent complexes, traces and so on. The underlying "metaresult" in its utmost generality is only stated at the end with details to be given in $\mathrm{BF}$.

We wish finally to thank the editorial committee of these proceedings for their inexhaustible patience awaiting completion of this paper.

\section{Chern Character and Griffiths' Transversality}

1.1. Let $Z \subseteq X$ be a codimension $q$ cycle on a compact Kähler manifold or a smooth quasi-projective variety $X$ defined over a field of characteristic zero. With $\Omega_{X}^{p}$ the sheaf of (holomorphic or algebraic) differential forms of order $p$ on $X$, the (cohomological) cycle or fundamental class of $Z$ in $\mathrm{H}^{q}\left(X, \Omega_{X}^{q}\right)$ has the following classical description due to Grothendieck [Gr1, p.151], see also [Mu, 2.18] for a recent treatment.

Let $c_{p}\left(\mathcal{O}_{Z}\right), \operatorname{ch}_{p}\left(\mathcal{O}_{Z}\right) \in \mathrm{H}^{p}\left(X, \Omega_{X}^{p}\right)$ denote the $p^{\text {th }}$ Chern class of $Z$ and the $p^{\text {th }}$ component of the Chern character of $Z$, respectively.

The fundamental (Hodge) class of $Z$ is

$$
[Z]=\frac{(-1)^{q-1} c_{q}\left(\mathcal{O}_{Z}\right)}{(q-1) !}=\operatorname{ch}_{q}\left(\mathcal{O}_{Z}\right) \in \mathrm{H}^{q}\left(X, \Omega_{X}^{q}\right) .
$$

Date: March 25, 2022.

R.O.B. was partly supported by NSERC grant 3-642-114-80.

Both authors were partly supported by a grant from the Volkswagen Foundation under the Research in Pairs program at Math. Forschungsinstitut Oberwolfach. 
As the components $\operatorname{ch}_{p}\left(\mathcal{O}_{Z}\right)$ vanish for $p$ below the codimension, the fundamental class is thus the leading term of the Chern character, $\operatorname{ch}\left(\mathcal{O}_{Z}\right)=\sum_{p \geq q} \operatorname{ch}_{p}\left(\mathcal{O}_{Z}\right) \in$ $\prod_{p} \mathrm{H}^{p}\left(X, \Omega_{X}^{p}\right)$.

1.2. Let $X$ be an analytic space or a scheme over a field. If $X$ is smooth, its first order (flat) deformations are parametrized by $\mathrm{H}^{1}\left(X, \Theta_{X}\right)$, the first cohomology group of the locally free sheaf of (holomorphic or algebraic) vector fields $\Theta_{X}=$ $\mathcal{H o m}_{X}\left(\Omega_{X}^{1}, \mathcal{O}_{X}\right)$.

The group $\mathrm{H}^{1}\left(X, \Theta_{X}\right) \cong \operatorname{Ext}_{X}^{1}\left(\Omega_{X}^{1}, \mathcal{O}_{X}\right)$ acts through graded derivations with respect to the total degree $p+q$ on the algebra $\prod_{p, q} \mathrm{H}^{q}\left(X, \Omega_{X}^{p}\right)$, sending a class of type $(p, q)$ to one of type $(p-1, q+1)$. Denoting the action of the class of a first order deformation $*$ on $c \in \mathrm{H}^{q}\left(X, \Omega_{X}^{p}\right)$ by $\left.*\right\lrcorner c$, as in [ALG, III.164, Prop. 10] or [Bld, 6.1], a codimension $q$ cycle $Z$ on $X$ defines thus a commutative diagram

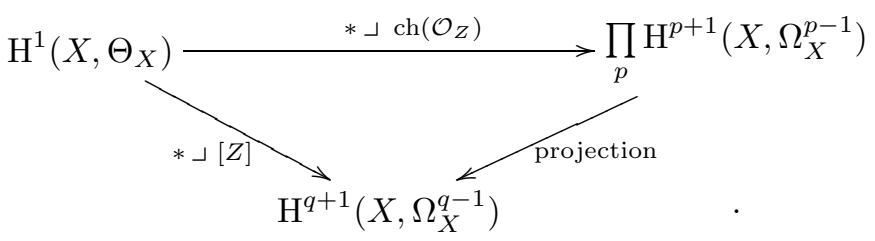

The geometric meaning of the action of first order deformations on cycle classes is explained by Bloch's interpretation of Griffiths' Transversality Theorem:

Theorem 1.3. ([Blo, (4.2)]; see [Voi, 2.3] for the analytic case.)

Given $\xi \in \mathrm{H}^{1}\left(X, \Theta_{X}\right)$, the unique horizontal lift of $[Z]$ relative to the Gauß-Manin connection defined by the infinitesimal deformation $\xi$ stays of type $(q, q)$ if and only if $\xi\lrcorner[Z]=0$.

One way of stating Grothendieck's (infinitesimal) Variational Hodge Conjecture Gr2], see also [Ste, §3], is to ask whether the obstruction expressed by Griffiths' Transversality is algebraic: If the unique horizontal lift of $[Z]$ along a first order deformation $\xi$ remains of Hodge type $(q, q)$, is there then some cycle representing the cycle class of $[Z]$ that deforms along $\xi$ in $X$ ? To begin with one may ask, as Bloch did in [Bld], what is the relationship between the obstruction to deform the given $Z$ along $\xi$ and the cohomological obstruction $\xi\lrcorner[Z]$ ?

This leads to the notion of semiregularity that we now recall.

\section{The Structure of the Hilbert Scheme and Semiregularity}

Let $X$ be an arbitrary analytic space or scheme over some field and let $Z \subseteq X$ be any closed subspace defined by a sheaf of ideals $\mathcal{I} \subseteq \mathcal{O}_{X}$. The embedded deformations of $Z \subseteq X$ are parametrized in the analytic case by the Douady space $H_{X}$ at the point corresponding to $Z \subseteq X$, and in the algebraic case by the Hilbert scheme Hilb $_{X}$ at $Z$.

2.1. The tangent space to $H_{X}$ or $\operatorname{Hilb}_{X}$ at the point corresponding to $Z$ is

$$
T_{Z / X}^{1}=\operatorname{Hom}_{Z}\left(\mathcal{I} / \mathcal{I}^{2}, \mathcal{O}_{Z}\right) \cong \mathrm{H}^{0}\left(Z, \mathcal{N}_{Z / X}\right)
$$

where $\mathcal{N}_{Z / X}$ is the normal sheaf to $Z$ in $X$. The obstruction space is contained in $T_{Z / X}^{2}$, the second cohomology group of the relative cotangent complex of $Z$ in $X$, 
see e.g. [11], [Rim, | Fle], |Pal]. One has the following inclusions and equalities that approximate this group and describe it in many situations:

$$
\begin{aligned}
\mathrm{H}^{1}\left(Z, \mathcal{N}_{Z / X}\right) & \subseteq \operatorname{Ext}_{Z}^{1}\left(\mathcal{I} / \mathcal{I}^{2}, \mathcal{O}_{Z}\right) & \subseteq & T_{Z / X}^{2} \\
\uparrow & & \uparrow & = \\
= & \text { if } Z \text { is locally } & & \text { if } Z \text { is generically a complete } \\
& \text { a complete intersection } & & \begin{array}{l}
\text { intersection and has no } \\
\text { embedded components. }
\end{array}
\end{aligned}
$$

2.2. With $X$ still an arbitrary space, denote $T_{X}^{1}$ its space of first order deformations. There is then a natural obstruction map ob $\operatorname{ob}_{Z X}: T_{X}^{1} \rightarrow T_{Z / X}^{2}$ so that $Z$ can be deformed along a given (first order) deformation $\xi$ of $X$ iff $\mathrm{ob}_{Z / X}(\xi)=0$.

If $X$ is smooth, then $T_{X}^{1} \cong \mathrm{H}^{1}\left(X, \Theta_{X}\right)$ and the obstruction map factors through the restriction map $\mathrm{H}^{1}\left(X, \Theta_{X}\right) \longrightarrow \mathrm{H}^{1}\left(Z, \Theta_{X} \otimes \mathcal{O}_{Z}\right) \cong \mathrm{H}^{1}\left(Z, \mathcal{H o m}{ }_{Z}\left(\Omega_{X}^{1} \otimes \mathcal{O}_{Z}, \mathcal{O}_{Z}\right)\right)$ followed by $\mathrm{H}^{1}(Z, \quad)$ of the $\mathcal{O}_{Z}$-dual of the Jacobi map $j: \mathcal{I} / \mathcal{I}^{2} \rightarrow \Omega_{X}^{1} \otimes \mathcal{O}_{Z}$,

$$
\mathrm{ob}_{Z / X}: T_{X}^{1} \cong \mathrm{H}^{1}\left(X, \Theta_{X}\right) \longrightarrow \mathrm{H}^{1}\left(Z, \Theta_{X} \otimes \mathcal{O}_{Z}\right) \stackrel{\mathrm{H}^{1}\left(Z, j^{*}\right)}{\longrightarrow} \mathrm{H}^{1}\left(Z, \mathcal{N}_{Z / X}\right) \subseteq T_{Z / X}^{2} .
$$

2.3. The question referred to above is now: Is there a natural semiregularity map

$$
\sigma: T_{Z / X}^{2} \longrightarrow \mathrm{H}^{q+1}\left(X, \Omega_{X}^{q-1}\right)
$$

such that the diagram

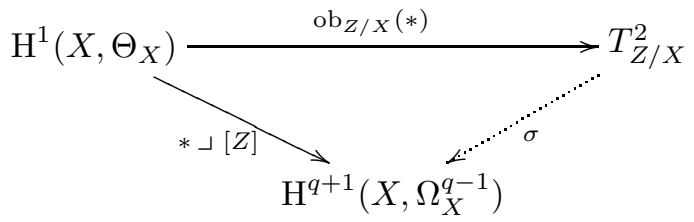

commutes?

2.4. If a semiregularity map exists, and if it is furthermore injective, then $Z$ is called semiregular in $X$.

Such a semiregularity map was first introduced by Severi [Sev| for a curve on a surface and he coined the term semiregular in that context. Kodaira-Spencer KSp] later generalized Severi's construction and results to arbitrary divisors on a complex manifold using the tools from the deformation theory they had just developed. Bloch $\mathrm{Bld}$ extended the notion to arbitrary cycles on smooth complex projective schemes, constructed a semiregularity map for cycles that are locally complete intersection, showed that the Hilbert scheme Hilb $\mathrm{b}_{X}$ is smooth at the point corresponding to a semiregular locally complete intersection $Z \subseteq X$, and deduced then the variational Hodge conjecture for the corresponding cycle class $[Z]$.

We will show here that a semiregularity map always exists, and that its construction could have been achieved more than 25 years ago, by anyone reading [Il] and $\mathrm{Blo}$ side by side!

The key ingredient is to forget part of the structure and to construct a more general semiregularity map for coherent sheaves on $X$. The underlying idea is simple: The fundamental class is not only defined for a cycle in $X$ but for any element of $K^{0}(X)$, the Grothendieck group of the smooth space $X$. Thus one may ask for a semiregularity map for any representative of a class in that group, that is 
for any coherent sheaf on $X$, or, if one allows $X$ to become singular, for any perfect complex of sheaves on $X$.

\section{Deformations of Modules and the Atiyah-Chern Character}

If $Z \subseteq X$ is a closed subspace of $X$, we may consider instead of embedded deformations of $Z$ the deformations of $\mathcal{O}_{Z}$ as $\mathcal{O}_{X}$-module. This is a forgetful functor: a deformation of $\mathcal{O}_{Z}$ as module will only be an invertible sheaf on its support, not necessarily its structure sheaf.

3.1. For any coherent sheaf $\mathcal{F}$ on $X$, the space of first order deformations is given by $\operatorname{Ext}_{X}^{1}(\mathcal{F}, \mathcal{F})$ with obstructions in $\operatorname{Ext}_{X}^{2}(\mathcal{F}, \mathcal{F})$, and so there are accordingly natural forgetful transformations

$$
f^{i}: T_{Z / X}^{i} \longrightarrow \operatorname{Ext}_{X}^{i}\left(\mathcal{O}_{Z}, \mathcal{O}_{Z}\right)
$$

These maps can be explained quite simply within the theory of resolvents and cotangent complexes, as developed in [Fl], [II], [Pa] based on [Qui]: a resolvent for $Z$ over $X$ is a differential graded free $\mathcal{O}_{X}$-algebra that resolves $\mathcal{O}_{Z}$, the groups $T_{Z / X}^{i}$ constitute the hypercohomology groups of the $\mathcal{O}_{X}$-derivations of said resolvent, whereas the groups $\operatorname{Ext}_{X}^{i}\left(\mathcal{O}_{Z}, \mathcal{O}_{Z}\right)$ represent the hypercohomology of its $\mathcal{O}_{X}$-linear endomorphisms. Now any $\mathcal{O}_{X}$-derivation is $\mathcal{O}_{X}$-linear by definition, whence the comparison maps; see [BF] for further details.

3.2. Note that $\operatorname{Ext}_{X}^{i}\left(\mathcal{O}_{Z}, \mathcal{O}_{Z}\right)$ always contains $\mathrm{H}^{i}\left(X, \mathcal{O}_{Z}\right)=\mathrm{H}^{i}\left(Z, \mathcal{O}_{Z}\right)$ naturally as a direct summand and that $f^{i}$ always maps into the complement. For $i=1$ one has indeed a split exact sequence

$$
0 \rightarrow T_{Z / X}^{1}=\mathrm{H}^{0}\left(X, \mathcal{N}_{Z / X}\right) \stackrel{f^{1}}{\longrightarrow} \operatorname{Ext}_{X}^{1}\left(\mathcal{O}_{Z}, \mathcal{O}_{Z}\right) \rightarrow \mathrm{H}^{1}\left(Z, \mathcal{O}_{Z}\right) \rightarrow 0
$$

and $\mathrm{H}^{1}\left(Z, \mathcal{O}_{Z}\right)$ is the tangent space to the Picard scheme of $Z$ at $\mathcal{O}_{Z}$. This formalizes that a first order deformation of $\mathcal{O}_{Z}$ as $\mathcal{O}_{X}$-module splits into an embedded deformation of $Z$ and a deformation of the invertible sheaf $\mathcal{O}_{Z}$ on $Z$.

When restricted to $\operatorname{Ext}_{Z}^{i-1}\left(\mathcal{I} / \mathcal{I}^{2}, \mathcal{O}_{Z}\right) \subseteq T_{Z / X}^{i}$, the forgetful maps $f^{i}$ become particularly explicit: The extension defined by the first fundamental neighbourhood of $Z$ in $X$,

$$
\mathcal{Z}^{(2)} \equiv 0 \longrightarrow \mathcal{I} / \mathcal{I}^{2} \longrightarrow \mathcal{O}_{X} / \mathcal{I}^{2} \longrightarrow \mathcal{O}_{Z} \longrightarrow 0
$$

defines a connecting homomorphism $\operatorname{Ext}_{X}^{i-1}\left(\mathcal{I} / \mathcal{I}^{2}, \mathcal{O}_{Z}\right) \rightarrow \operatorname{Ext}_{X}^{i}\left(\mathcal{O}_{Z}, \mathcal{O}_{Z}\right)$ which composed with the forgetful map $\operatorname{Ext}_{Z}^{i-1}\left(\mathcal{I} / \mathcal{I}^{2}, \mathcal{O}_{Z}\right) \rightarrow \operatorname{Ext}_{X}^{i-1}\left(\mathcal{I} / \mathcal{I}^{2}, \mathcal{O}_{Z}\right)$ yields the restriction of $f^{i}$.

3.3. Returning to the context of coherent $\mathcal{O}_{X}$-modules on a smooth space $X$ we want thus to construct a general semiregularity map for coherent $\mathcal{O}_{X}$-modules $\mathcal{F}$,

$$
\tau^{2}=\left(\tau^{2, p}\right)_{p \geq 0}: \operatorname{Ext}_{X}^{2}(\mathcal{F}, \mathcal{F}) \longrightarrow \prod_{p \geq 0} \mathrm{H}^{p+2}\left(X, \Omega_{X}^{p}\right)
$$

such that the desired semiregularity map for a cycle $Z$ of codimension $q$ will be

$$
\sigma=\tau^{2, q-1} f^{2}: T_{Z / X}^{2} \longrightarrow \operatorname{Ext}_{X}^{2}\left(\mathcal{O}_{Z}, \mathcal{O}_{Z}\right) \longrightarrow \mathrm{H}^{q+1}\left(X, \Omega_{X}^{q-1}\right) .
$$

A natural such map $\tau$ has been known for a long time! 
3.4. To describe it, recall the definition of the Atiyah class of an $\mathcal{O}_{X}$-module $\mathcal{F}$ : Let $X^{(2)} \subseteq X \times X$ denote the first fundamental neighbourhood of the diagonal $\Delta: X \hookrightarrow X \times X$ and denote $p_{i}: X^{(2)} \rightarrow X$ the projection onto the $i^{\text {th }}$ factor. The sheaf of 1 -jets or principal parts of first order associated to $\mathcal{F}$ is defined to be $\mathcal{P}^{1}(F)=p_{2 *} p_{1}^{*} \mathcal{F}$. Restricting $\mathcal{P}^{1}(F)$ to the diagonal itself defines a canonical exact sequence of (right) $\mathcal{O}_{X}$-modules, the Atiyah sequence of $\mathcal{F}$,

$$
\operatorname{At}_{X}(\mathcal{F}) \equiv 0 \longrightarrow \mathcal{F} \otimes \Omega_{X}^{1} \longrightarrow \mathcal{P}^{1}(\mathcal{F}) \longrightarrow \mathcal{F} \longrightarrow 0
$$

whose class

$$
\operatorname{at}_{X}(\mathcal{F}) \in \operatorname{Ext}_{X}^{1}\left(\mathcal{F}, \mathcal{F} \otimes \Omega_{X}^{1}\right)
$$

is the (first) Atiyah class of $\mathcal{F}$.

3.5. Combining the composition or Yoneda product with the exterior product on $\Omega_{X}^{*}$ defines on the vector space $\prod_{i, j} \operatorname{Ext}_{X}^{i}\left(\mathcal{F}, \mathcal{F} \otimes \Omega_{X}^{j}\right)$ an algebra structure with multiplication

$$
\smile: \operatorname{Ext}_{X}^{a}\left(\mathcal{F}, \mathcal{F} \otimes \Omega_{X}^{b}\right) \times \operatorname{Ext}_{X}^{c}\left(\mathcal{F}, \mathcal{F} \otimes \Omega_{X}^{d}\right) \longrightarrow \operatorname{Ext}_{X}^{a+c}\left(\mathcal{F}, \mathcal{F} \otimes \Omega_{X}^{b+d}\right) .
$$

One can thus form powers of the Atiyah class,

$$
\operatorname{at}_{X}^{p}(\mathcal{F})=\operatorname{at}_{X}(\mathcal{F})^{\smile p} \in \operatorname{Ext}_{X}^{p}\left(\mathcal{F}, \mathcal{F} \otimes \Omega_{X}^{p}\right),
$$

and then, in characteristic zero, the Atiyah-Chern character

$$
e^{\operatorname{at}_{X}(\mathcal{F})}=\sum_{p \geq 0} \frac{\operatorname{at}_{X}^{p}(\mathcal{F})}{p !} \in \prod_{p} \operatorname{Ext}_{X}^{p}\left(\mathcal{F}, \mathcal{F} \otimes \Omega_{X}^{p}\right) .
$$

The (powers of the) Atiyah class and the Atiyah-Chern character are functorial in the appropriate sense; in particular these notions localize to yield local sections in $\prod_{p} \mathcal{E} x t_{X}^{p}\left(\mathcal{F}, \mathcal{F} \otimes \Omega_{X}^{p}\right)$. Various explicit representatives of these extension classes are worked out in [AL.] in the algebraic case.

3.6. As $X$ is supposed to be smooth, any coherent $\mathcal{O}_{X}$-module $\mathcal{F}$ admits locally a finite resolution by locally free sheaves, that is, it represents a perfect complex, and thus there exist natural trace maps

$$
\operatorname{tr}^{p}: \operatorname{Ext}_{X}^{p}\left(\mathcal{F}, \mathcal{F} \otimes \Omega_{X}^{p}\right) \rightarrow \mathrm{H}^{p}\left(X, \Omega_{X}^{p}\right)
$$

that uniquely extend the usual trace $\operatorname{tr}^{0}$ of an endomorphism of $\mathcal{F}$. The quite intricate technicalities in the general setting were overcome in the algebraic case by Illusie, first in SGA 6, Exp. I], then in [II], and the analytic case was dealt with explicitly by O'Brian, Toledo, Tong in OTT.

It is a classical result that the trace of (the inverse of) the Atiyah-Chern character yields the Chern character:

Theorem 3.7. (Atiyah [At]; Illusie [II]; O'Brian-Toledo-Tong [OTT]) If $\mathcal{F}$ is a coherent sheaf on the smooth space $X$, then

$$
\operatorname{ch}(\mathcal{F})=\operatorname{tr}\left(e^{-\operatorname{at}_{X}(\mathcal{F})}\right) \in \prod_{p} \mathrm{H}^{p}\left(X, \Omega_{X}^{p}\right) .
$$

In particular, for a closed subspace $Z \subseteq X$ of codimension $q$, one has

$$
[Z]=\operatorname{ch}_{q}\left(\mathcal{O}_{Z}\right)=\frac{(-1)^{q} \operatorname{tr}\left(\operatorname{at}_{X}^{q}\left(\mathcal{O}_{Z}\right)\right)}{q !} .
$$


The minus sign appears here as traditionally the first Chern class is the trace of the opposite of the Atiyah class, see [At, Prop.12], [I1], V.5.4.1, 5.9.4]. Note also that extension to the algebraic case removed the factor $2 \pi i$ originally found in the exponent.

The link between this theorem and the existence of a semiregularity map is the following result, due to Illusie [II], IV.3.1.8] in much vaster generality.

Proposition 3.8. If $\mathcal{F}$ is a coherent sheaf on the smooth space $X$, then the obstruction $\operatorname{ob}_{\mathcal{F}}: T_{X}^{1} \rightarrow \operatorname{Ext}_{X}^{2}(\mathcal{F}, \mathcal{F}$ ) to deform $\mathcal{F}$ (flatly) along a given first order deformation of $X$ can be realized as

$\operatorname{ob}_{\mathcal{F}}: T_{X}^{1} \cong \operatorname{Ext}_{X}^{1}\left(\Omega_{X}^{1}, \mathcal{O}_{X}\right) \stackrel{\mathcal{F} \otimes()}{\longrightarrow} \operatorname{Ext}_{X}^{1}\left(\mathcal{F} \otimes \Omega_{X}^{1}, \mathcal{F}\right) \stackrel{(~) \circ\left(-\operatorname{at}_{X}(\mathcal{F})\right)}{\longrightarrow} \operatorname{Ext}_{X}^{2}(\mathcal{F}, \mathcal{F})$.

In comparison to Illusie's original treatment we changed here the sign in front of the Atiyah class to incur fewer signs later on.

3.9. The obstructions for embedded deformations of a subspace $Z$ versus those for deformations of the module $\mathcal{O}_{Z}$ are related explicitly by means of the following commutative diagram whose rows are exact sequences of $\mathcal{O}_{X}$-modules, namely the Atiyah sequence of the $\mathcal{O}_{X}$-module $\mathcal{O}_{Z}$ and the exact sequence of the first infinitesimal neighbourhood of $Z$ in $X$, respectively:

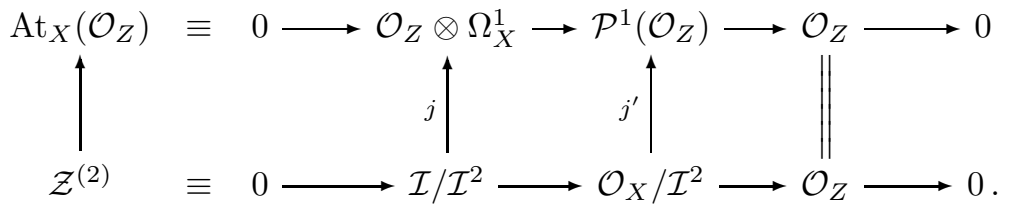

The map $j^{\prime}$ is given by $j^{\prime}(x)=1 \otimes p_{2}^{*}(x) \in \mathcal{P}^{1}\left(\mathcal{O}_{Z}\right)=p_{1}^{*} \mathcal{O}_{Z} \otimes\left(\mathcal{O}_{X \times X} / \mathcal{I}_{\Delta}^{2}\right)$, where $x$ is a local section of $\mathcal{O}_{X} / \mathcal{I}^{2}$ and $\mathcal{I}_{\Delta}$ denotes the ideal of the diagonal in $X \times X$. Indeed, for a local section $f \in \mathcal{I}$ one has that $j(f)$ lifts to

$$
\begin{aligned}
\tilde{j}(f) & =1 \otimes d f \in \mathcal{O}_{X} \otimes \Omega_{X}^{1} \\
& \mapsto\left(p_{2}^{*}(f)-p_{1}^{*}(f)\right)=1 \otimes f-f \otimes 1 \in \mathcal{O}_{X \times X} / \mathcal{I}_{\Delta}^{2} \\
& \mapsto 1 \otimes p_{2}^{*}(f) \in p_{1}^{*} \mathcal{O}_{Z} \cong \mathcal{O}_{Z \times X} / \mathcal{O}_{Z \times X} \mathcal{I}_{\Delta}^{2} \\
& \mapsto j^{\prime}(f) \in \mathcal{P}^{1}\left(\mathcal{O}_{Z}\right) .
\end{aligned}
$$

In view of (3.2) this means that indeed $\mathrm{ob}_{\mathcal{O}_{Z}}=f^{2}{ }_{\circ} \mathrm{ob}_{Z / X}$ relates the obstruction maps of the two deformation problems.

Now we are ready to define

\section{The Generalized Semiregularity MaP}

4.1. The homomorphism of $\mathcal{O}_{X}$-algebras $\mathcal{O}_{X} \rightarrow \Omega_{X}^{*}$ induces an algebra homomorphism

$$
\prod_{i} \operatorname{Ext}_{X}^{i}(\mathcal{F}, \mathcal{F}) \longrightarrow \prod_{i, j} \operatorname{Ext}_{X}^{i}\left(\mathcal{F}, \mathcal{F} \otimes \Omega_{X}^{j}\right)
$$

which extends the natural right action of the Yoneda Ext-algebra $\operatorname{Ext}_{X}(\mathcal{F}, \mathcal{F})$ on the target. Contraction combined with Yoneda product defines further a left action

$$
\operatorname{Ext}_{X}^{r}\left(\Omega_{X}^{s}, \mathcal{O}_{X}\right) \times \operatorname{Ext}_{X}^{i}\left(\mathcal{F}, \mathcal{F} \otimes \Omega_{X}^{j}\right) \stackrel{\lrcorner}{\longrightarrow} \operatorname{Ext}_{X}^{r+i}\left(\mathcal{F}, \mathcal{F} \otimes \Omega_{X}^{j-s}\right)
$$


and its restriction to $\operatorname{Ext}_{X}^{1}\left(\Omega_{X}^{1}, \mathcal{O}_{X}\right)$ acts through graded algebra derivations with respect to total degree,

$$
\left.\left.\xi\lrcorner\left(\omega \smile \omega^{\prime}\right)=(\xi\lrcorner \omega\right) \smile \omega^{\prime}+(-1)^{i+j} \omega \smile(\xi\lrcorner \omega^{\prime}\right),
$$

for $\xi \in \operatorname{Ext}_{X}^{1}\left(\Omega_{X}^{1}, \mathcal{O}_{X}\right), \omega \in \operatorname{Ext}_{X}^{i}\left(\mathcal{F}, \mathcal{F} \otimes \Omega_{X}^{j}\right)$.

4.2. Combining these actions, one obtains for any coherent sheaf $\mathcal{F}$ a family of generalized semiregularity maps,

$$
\tau^{i}=\left(\tau^{i, p}\right)_{p \geq 0}=\operatorname{tr}\left(e^{-\operatorname{at}_{X}(\mathcal{F})} \smile *\right): \operatorname{Ext}_{X}^{i}(\mathcal{F}, \mathcal{F}) \longrightarrow \prod_{p} \mathrm{H}^{i+p}\left(X, \Omega_{X}^{p}\right)
$$

that is natural in $\mathcal{F}$ and whose value on $\operatorname{id}_{\mathcal{F}} \in \operatorname{Ext}_{X}^{0}(\mathcal{F}, \mathcal{F})$ is the Chern character of $\mathcal{F}$.

4.3. If $Z \subseteq X$ contains the support of $\mathcal{F}$, then the trace map factors through the local cohomology with support in $Z$, see [III, V.6] or [OTT, 4.6], whence one may define Chern character and generalized semiregularity maps with support,

$$
\begin{aligned}
\operatorname{ch}_{Z}(\mathcal{F}) & =\operatorname{tr}_{Z}\left(e^{-\operatorname{at}_{X}(\mathcal{F})}\right) \in \prod_{p} \mathrm{H}_{Z}^{p}\left(X, \Omega_{X}^{p}\right) \\
\tau_{Z} & =\operatorname{tr}_{Z}\left(e^{-\operatorname{at}_{X}(\mathcal{F})} \smile *\right): \prod_{i} \operatorname{Ext}_{X}^{i}(\mathcal{F}, \mathcal{F}) \longrightarrow \prod_{i, p} \mathrm{H}_{Z}^{i+p}\left(X, \Omega_{X}^{p}\right) .
\end{aligned}
$$

The key result is now the following.

Theorem 4.4. A coherent $\mathcal{O}_{X}$-module $\mathcal{F}$ on a smooth space $X$ whose support is contained in $Z \subseteq X$ gives rise to a commutative diagram

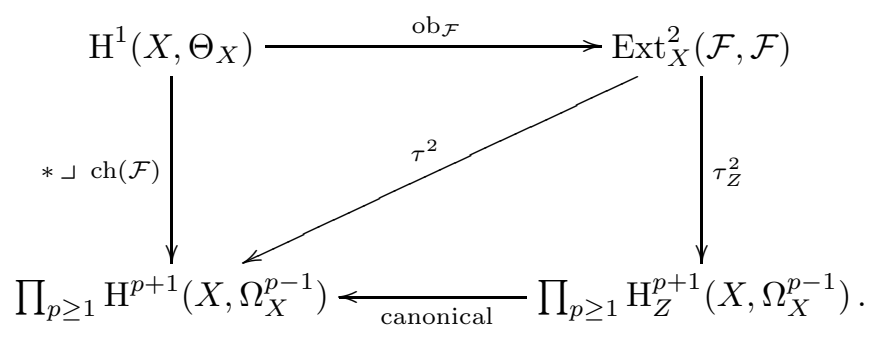

Proof. Illusie's result (3.8) shows that $\left.\mathrm{ob}_{\mathcal{F}}(\xi)=\xi\right\lrcorner-\mathrm{at}_{X}(\mathcal{F})$ for any first order deformation $\xi \in \mathrm{H}^{1}\left(X, \Theta_{X}\right)$. The class at $\operatorname{tat}_{X}(\mathcal{F})$ is of total degree two, and so

$$
\begin{aligned}
& \xi\lrcorner \operatorname{ch}(\mathcal{F})=\xi\lrcorner \operatorname{tr}\left(e^{-\operatorname{at}_{X}(\mathcal{F})}\right) \\
& \left.=\operatorname{tr}(\xi\lrcorner e^{-\operatorname{at}_{X}(\mathcal{F})}\right) \quad \text { by naturality of the trace, } \\
& \left.=\operatorname{tr}\left(e^{-\operatorname{at}_{X}(\mathcal{F})} \smile(\xi\lrcorner-\operatorname{at}_{X}(\mathcal{F})\right)\right) \\
& \text { as } \operatorname{tr}(a b)=\operatorname{tr}(b a) \text { for even classes } a \text { and } b \text {, whence } \\
& \operatorname{tr}\left(D\left(e^{-y}\right)\right)=\operatorname{tr}\left(e^{-y} D(-y)\right) \text { for any (graded) } \\
& \text { derivation } D \text { and any (even) element } y \text {, }
\end{aligned}
$$

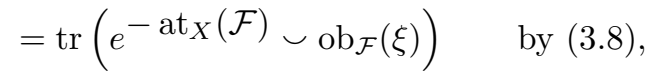

$$
\begin{aligned}
& =\tau^{2}\left(\operatorname{ob}_{\mathcal{F}}(\xi)\right) \text {. }
\end{aligned}
$$


Factoring the obstruction map ob $\mathcal{O}_{Z}$ through $f^{2}$ gives now the desired generalized semiregularity map for cycles.

Theorem 4.5. If $Z \subseteq X$ is a closed (analytic or algebraic) subspace of the smooth space $X$, then the diagram

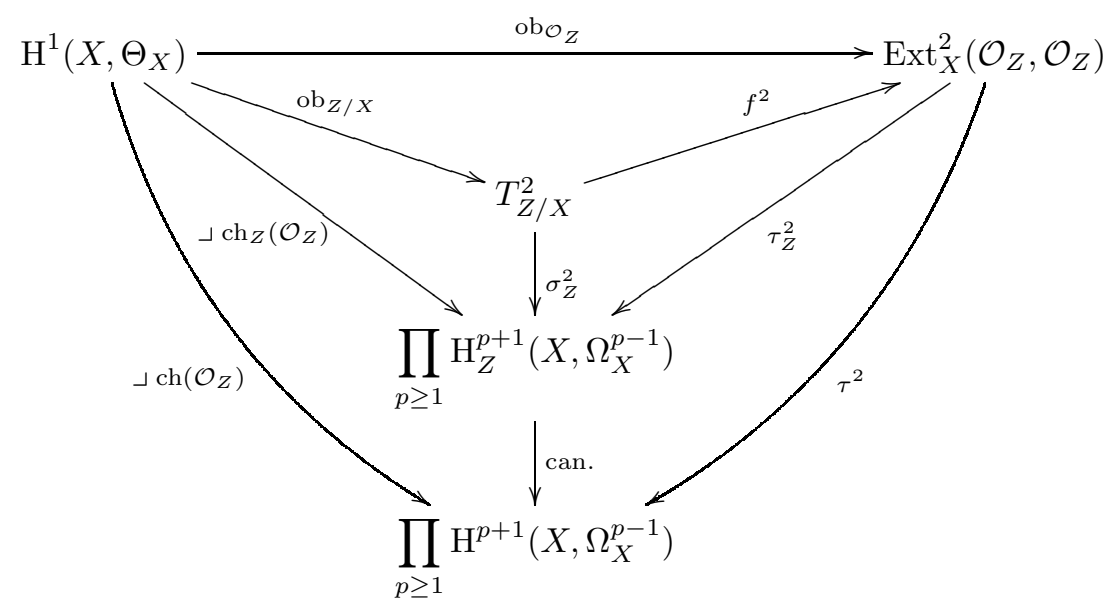

commutes, where we define $\sigma_{Z}^{2}=\tau_{Z}^{2} \cdot f^{2}$.

If $Z$ is of codimension $q$, then the component $\sigma_{Z}^{2, q-1}=\tau_{Z}^{2, q-1} \cdot f^{2}$ is a semiregularity map with support for $Z$, whereas its composition with the canonical map yields the desired (absolute) semiregularity map

$$
\sigma^{2, q-1}=\tau^{2} \cdot f^{2}: T_{Z / X}^{2} \longrightarrow \mathrm{H}^{q+1}\left(X, \Omega_{X}^{q-1}\right) .
$$

4.6. More generally, we can define as well a family of generalized semiregularity maps for $Z$ (with support) through

$$
\sigma_{(Z)}^{i}=\tau_{(Z)}^{i} \cdot f^{i}: T_{Z / X}^{i} \longrightarrow \prod_{p} \mathrm{H}_{(Z)}^{i+p}\left(X, \Omega_{X}^{p}\right) .
$$

\section{Duality and the Infinitesimal Abel-Jacobi MaP}

5.1. Due to their naturality, the trace maps with support as well as the (powers of the) Atiyah class localize to yield generalized semiregularity maps on the level of sheaves

$$
\tilde{\tau}^{i}: \mathcal{E} x t_{X}^{i}(\mathcal{F}, \mathcal{F}) \rightarrow \prod_{p} \mathcal{H}_{Z}^{i+p}\left(X, \Omega_{X}^{p}\right)
$$

and these morphisms of sheaves fit together to morphisms of the associated localglobal spectral sequences

$$
\begin{gathered}
\mathrm{H}^{i}\left(X, \mathcal{E} x t_{X}^{j}(\mathcal{F}, \mathcal{F})\right) \Longrightarrow \operatorname{Ext}_{X}^{i+j}(\mathcal{F}, \mathcal{F}) \\
\mathrm{H}^{i}\left(X, \tilde{\tau}^{j}\right) \downarrow \\
\prod_{p} \mathrm{H}^{i}\left(X, \mathcal{H}_{Z}^{p+j}\left(X, \Omega_{X}^{p}\right)\right) \Longrightarrow \prod_{p} \mathrm{H}_{Z}^{i+j+p}\left(X, \Omega_{X}^{p}\right) .
\end{gathered}
$$


As $X$ is assumed to be smooth, each $\Omega_{X}^{p}$ is locally free and $\mathcal{H}_{Z}^{k}\left(X, \Omega_{X}^{p}\right)=0$ for $k<\operatorname{depth}_{Z} \mathcal{O}_{X}$. It follows that the components $\tau_{Z}^{i, p}$, and then a fortiori $\tau^{i, p}$ and $\sigma_{Z}^{i, p}$ or $\sigma^{i, p}$ for $Z$, vanish whenever $i+p<\operatorname{depth}_{Z} \mathcal{O}_{X}$.

5.2. If $Z \subseteq X$ is a closed subspace of codimension $q$ that is locally Cohen-Macaulay, then the local cohomology sheaves $\mathcal{H}_{Z}^{q^{\prime}}\left(X, \Omega_{X}^{p}\right)$ vanish for $q^{\prime}<q$ and any $p$, and so there are natural inclusions $\mathrm{H}^{i}\left(X, \mathcal{H}_{Z}^{q}\left(X, \Omega_{X}^{p}\right)\right) \hookrightarrow \mathrm{H}_{Z}^{i+q}\left(X, \Omega_{X}^{p}\right)$.

Now assume that the given subspace satisfies furthermore $T_{Z / X}^{2}=\mathrm{H}^{1}\left(X, \mathcal{N}_{Z / X}\right)$. The isomorphisms of $\mathcal{O}_{X}$-modules

$$
\mathcal{N}_{Z / X} \cong \mathcal{H o m}_{Z}\left(\mathcal{I} / \mathcal{I}^{2}, \mathcal{O}_{Z}\right) \cong \mathcal{H o m}_{X}\left(\mathcal{I}, \mathcal{O}_{Z}\right) \cong \mathcal{E} t_{X}^{1}\left(\mathcal{O}_{Z}, \mathcal{O}_{Z}\right)
$$

show that the semiregularity maps with support, $\sigma_{Z}^{2, q-1}: T_{Z / X}^{2} \rightarrow \mathrm{H}_{Z}^{q+1}\left(X, \Omega_{X}^{q-1}\right)$, factors through $\mathrm{H}^{1}\left(X, \tilde{\sigma}_{Z}^{1, q-1}\right)$. Thus the semiregularity map $\sigma^{2, q-1}$ itself fits into the following commutative diagram

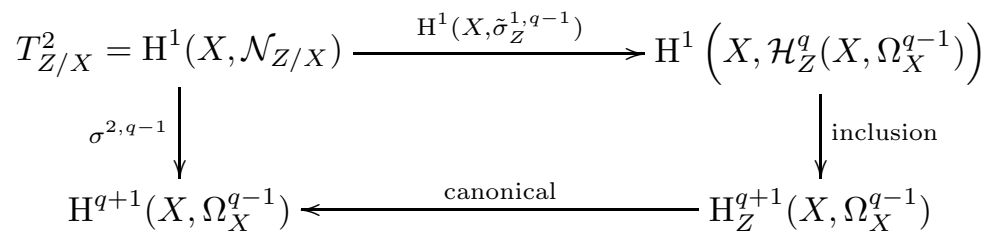

5.3. If $Z$ is locally a complete intersection of codimension $q$ in $X$, it is in particular locally Cohen-Macaulay and satisfies $T_{Z / X}^{2}=\mathrm{H}^{1}\left(X, \mathcal{N}_{Z / X}\right)$. With $d=\operatorname{dim} Z$ and $\mathcal{I}$ the defining ideal of $Z$, take exterior powers of the Jacobi map $j: \mathcal{I} / \mathcal{I}^{2} \rightarrow \Omega_{X}^{1} \otimes \mathcal{O}_{Z}$ to define for $0 \leq i \leq q$ natural pairings

$$
\Omega_{X}^{d+i} \otimes \Lambda^{q-i}\left(\mathcal{I} / \mathcal{I}^{2}\right) \stackrel{1 \otimes \Lambda^{q-i} j}{\longrightarrow} \Omega_{X}^{d+i} \otimes \Omega_{X}^{q-i} \otimes \mathcal{O}_{Z} \stackrel{(\wedge) \otimes 1}{\longrightarrow} \omega_{X} \otimes \mathcal{O}_{Z} .
$$

As $\mathcal{I} / \mathcal{I}^{2}$ is locally free, these give rise, equivalently, to $\mathcal{O}_{X}$-homomorphisms

$$
\gamma_{i}: \Omega_{X}^{d+i} \longrightarrow \omega_{X} \otimes \Lambda^{q-i} \mathcal{N}_{Z / X} \cong \omega_{Z} \otimes \Lambda^{i}\left(\mathcal{I} / \mathcal{I}^{2}\right)
$$

where we have used the adjunction formula $\omega_{Z} \cong \omega_{X} \otimes \operatorname{det} \mathcal{N}_{Z / X}$ for the dualizing module $\omega_{Z}$, and the isomorphism $\Lambda^{q-i} \mathcal{N}_{Z / X} \cong \operatorname{det} \mathcal{N}_{Z / X} \otimes \Lambda^{i}\left(\mathcal{I} / \mathcal{I}^{2}\right)$.

The duality theorem for finite morphisms says that

$$
\operatorname{Ext}_{X}^{p}\left(\omega_{Z} \otimes \Lambda^{i}\left(\mathcal{I} / \mathcal{I}^{2}\right), \omega_{X}\right) \cong \operatorname{Ext}_{Z}^{p-q}\left(\Lambda^{i}\left(\mathcal{I} / \mathcal{I}^{2}\right), \mathcal{O}_{Z}\right) \cong \mathrm{H}^{p-q}\left(Z, \Lambda^{i} \mathcal{N}_{Z / X}\right)
$$

and clearly

$$
\operatorname{Ext}_{X}^{p}\left(\Omega_{X}^{d+i}, \omega_{X}\right) \cong \mathrm{H}^{p}\left(X, \Omega_{X}^{q-i}\right)
$$

for any $p$ or $i$, whence $\check{\gamma}^{i, p}=\operatorname{Ext}_{X}^{p}\left(\gamma_{i}, \omega_{X}\right)$ can be identified as a map between cohomology groups,

$$
\check{\gamma}^{i, p}: \mathrm{H}^{p-q}\left(Z, \Lambda^{i} \mathcal{N}_{Z / X}\right) \longrightarrow \mathrm{H}^{p}\left(X, \Omega_{X}^{q-i}\right) .
$$

Equivalently, $\check{\gamma}^{i, p}$ is the Serre dual of the map $\mathrm{H}_{c}^{d+q-p}\left(X, \Omega_{X}^{d+i}\right) \rightarrow \mathrm{H}_{c}^{d+q-p}\left(Z, \omega_{Z} \otimes\right.$ $\left.\Lambda^{i} \mathcal{I} / \mathcal{I}^{2}\right)$ induced by $\gamma_{i}$.

5.4. Some of these maps are classical objects: 
1. For $i=0, p=q$, and with $f: Z \hookrightarrow X$ the closed embedding, one finds by [FGA, 149-20, Thm.4] the leading term of the direct image map $f_{*}$ in cohomology,

$$
\check{\gamma}^{0, q}=\left.f_{*}\right|_{\mathrm{H}^{0}\left(Z, \mathcal{O}_{Z}\right)}: \mathrm{H}^{0}\left(Z, \mathcal{O}_{Z}\right) \longrightarrow \mathrm{H}^{q}\left(X, \Omega_{X}^{q}\right) .
$$

By definition, $\check{\gamma}^{0, q}\left(1_{Z}\right)=[Z]$, the fundamental (Hodge) class of $Z$, and a simple application of the Grothendieck-Riemann-Roch Theorem, see Mur, 2.11], shows $\check{\gamma}^{0, q}\left(1_{Z}\right)=\operatorname{ch}_{q}\left(\mathcal{O}_{Z}\right)$ which is the result quoted at the beginning (1.1).

2. For $i=1, p=q$ and $Z$ smooth on a compact Kähler manifold, the map $\check{\gamma}^{1, q}$ is the infinitesimal Abel-Jacobi map at $Z$ as shown in [Gre, Lect.2, p.29],

$$
\check{\gamma}^{1, q}=A J_{X, Z}: \mathrm{H}^{0}\left(X, \mathcal{N}_{Z / X}\right) \longrightarrow \mathrm{H}^{q}\left(X, \Omega_{X}^{q-1}\right) .
$$

3. For $i=1, p=q-1$ and $Z$ again locally a complete intersection, one finds Bloch's semiregularity map

$$
\check{\gamma}^{1, q+1}=\sigma_{\text {Bloch }}: \mathrm{H}^{1}\left(Z, \mathcal{N}_{Z / X}\right) \longrightarrow \mathrm{H}^{q+1}\left(X, \Omega_{X}^{q-1}\right) .
$$

These observations lead to the following duality theorem for the leading terms of the generalized semiregularity maps.

Theorem 5.5. Let $Z \subseteq X$ be a closed subspace of codimension $q$ that is locally a complete intersection.

(1) One has $T_{Z / X}^{p} \cong \mathrm{H}^{p-1}\left(Z, \mathcal{N}_{Z / X}\right)$ and

$$
\sigma^{p, q-1} \cong \check{\gamma}^{1, p+q-1}: T_{Z / X}^{p} \longrightarrow \mathrm{H}^{p+q-1}\left(X, \Omega_{X}^{q-1}\right) .
$$

Each of these maps fits into a commutative diagram

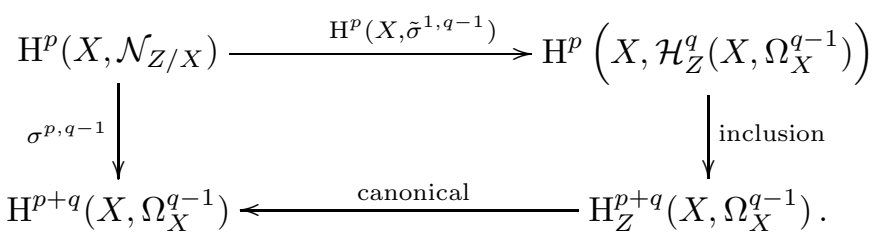

(2) Bloch's semiregularity map satisfies $\sigma_{\mathrm{Bloch}}=\sigma^{2, q-1}$.

(3) If $Z$ is smooth in a compact Kähler manifold $X$, then the infinitesimal AbelJacobi map at $Z$ is given by the component $\sigma^{1, q-1}$ of the generalized semiregularity map.

Clearly $(1) \Longrightarrow(2),(3)$ in view of the explicit construction of both $\sigma_{\text {Bloch }}$ and $A J_{X, Z}$. Verification of (1) is achieved through a local calculation that identifies $\tilde{\sigma}^{1, q-1}$. The patient reader is referred to the details in [BF] whereas the impatient one may wish to extract the calculation from the explicit formalism in [ALJ].

5.6. Of course, this result raises several questions, in particular whether for any algebraic cycle $Z$ on a smooth space the diagram

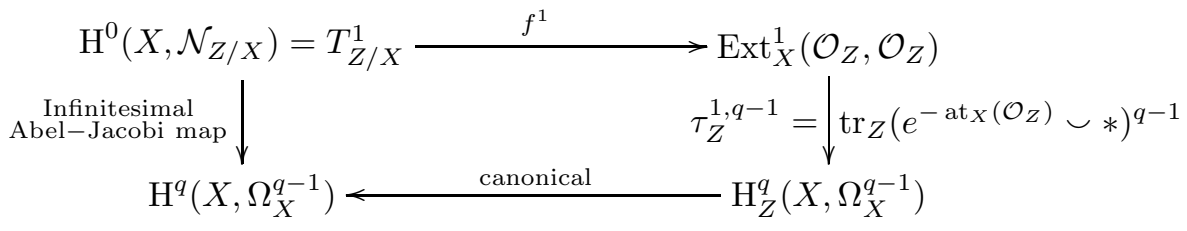


commutes. Note that $f^{1}$ is always a split monomorphism by (3.2) so that the general infinitesimal Abel-Jacobi map should be a retract of $\tau^{1, q-1}$.

Even more generally, there should be an Abel-Jacobi map for deformations of arbitrary coherent sheaves on a compact algebraic or Kähler manifold such that its differential is essentially given by the trace applied to multiplication with powers of the Atiyah class.

\section{Applications to Deformation Theory}

6.1. Ideally, one would like to interpret the family of semiregularity maps $\sigma$ or $\tau$ as maps between cohomology groups of cotangent complexes induced by morphisms of deformation theories. Whereas the domain of these maps is of the desired form, it is not clear what kind of deformation theory, if any, would allow the groups $\mathbb{H}^{q}:=\prod_{p} \mathrm{H}^{p+q}\left(X, \Omega_{X}^{p}\right)$ as its tangent cohomology. Hodge theory shows that if such a deformation theory existed, it were necessarily non obstructed for any complex manifold $X$ that is bimeromorphically equivalent to a Kähler manifold: According to Deligne [De], (5.5)], each of the functors

$$
\mathcal{N} \mapsto \mathrm{H}^{q}\left(X_{T}, \Omega_{X_{T} / T}^{p} \otimes_{\mathcal{O}_{T}} \mathcal{N}\right)
$$

on the coherent modules $\mathcal{N}$ over an artinian complex germ $T$ is then exact, and this applied to $\mathbb{H}^{1}$ would show that every "infinitesimal deformation" could be lifted. This line of reasoning applies indeed directly in some special cases, see Ran4.

In general, even though there might not be an actual deformation theory underlying the groups $\mathbb{H}^{q}$, it was first pointed out by Ran, [Ran1, Ran2, $\left.\operatorname{Ran} 3\right]$, and then made more precise in Kaw1, Kaw2, Kaw3 and [FMa], that Deligne's theorem can be used to obtain results on embedded deformations or deformations of coherent modules. Complete proofs and further generalizations of the following results will be contained in $[\mathrm{BF}]$.

Theorem 6.2. Assume that $f: X \rightarrow \Sigma$ is a smooth morphism of complex spaces with $X_{0}:=f^{-1}(0)$ a compact manifold bimeromorphically equivalent to a Kähler manifold. Let $\mathcal{F}_{0}$ be a coherent module on $X_{0}$ and

$$
\tau^{2}=\operatorname{tr}\left(e^{-\operatorname{at}\left(\mathcal{F}_{0}\right)} \smile *\right): \operatorname{Ext}_{X_{0}}^{2}\left(\mathcal{F}_{0}, \mathcal{F}_{0}\right) \longrightarrow \prod_{p \geq 0} H^{p+2}\left(X_{0}, \Omega_{X_{0}}^{p}\right)
$$

the corresponding generalized semiregularity map. The dimension of the basis $S$ of a semi-universal deformation of $\mathcal{F}_{0}$ satisfies

$$
\operatorname{dim} S \geq \operatorname{dim}_{\mathbb{C}} \operatorname{Ext}_{X_{0}}^{1}\left(\mathcal{F}_{0}, \mathcal{F}_{0}\right)-\operatorname{dim}_{\mathbb{C}} \operatorname{ker} \tau^{2} .
$$

If $\tau^{2}$ is injective then $S$ is smooth over a closed subspace of $\Sigma$.

6.3. This result generalizes in characteristic zero the Artamkin-Mukai criterion Art, Muk, Kaw1 which is the above statement but restricted to the component $\tau^{2,0}: \operatorname{Ext}_{X}^{2}(\mathcal{F}, \mathcal{F}) \longrightarrow H^{2}\left(X, \mathcal{O}_{X}\right)$ in the absolute case where $X=X_{0}$ is a nonsingular projective variety. The map $\tau^{2,0}$ is just the ordinary trace and this component of the generalized semiregularity map has indeed a description as a transformation between tangent cohomologies induced by a morphism of deformation theories: As $X$ is smooth, every coherent sheaf $\mathcal{F}$ admits a well defined determinant line bundle $\operatorname{det} \mathcal{F}$, and associating to $\mathcal{F}$ its determinant is a morphism of deformation functors that induces $\tau^{i, 0}: \operatorname{Ext}_{X}^{i}(\mathcal{F}, \mathcal{F}) \longrightarrow H^{i}\left(X, \mathcal{O}_{X}\right)$ on the corresponding tangent cohomology groups. 
As the target, the Picard functor of $X$, is smooth, it follows that all obstructions to deform $\mathcal{F}$ are already contained in the kernel of the trace map $\tau^{2,0}$. This deformation theoretic interpretation allows Artamkin to obtain as well results in positive characteristic that are beyond reach here.

The analogous application to embedded deformations of subspaces generalizes Bloch's original result [Bld, Thm.(7.3)] relating semiregularity and smoothness of the Hilbert scheme. It encompasses the previous generalizations of that result in Ran2, Kaw1.

Theorem 6.4. Assume that $f: X \rightarrow \Sigma$ is a smooth morphism of complex spaces and that $X_{0}:=f^{-1}(0)$ is a compact manifold bimeromorphically equivalent to a Kähler manifold. Let $Z \subseteq X_{0}$ be a closed subspace and

$$
\sigma^{2}: T_{Z / X_{0}}^{2}\left(\mathcal{O}_{Z}\right) \longrightarrow \prod_{p \geq 0} H^{p+2}\left(X_{0}, \Omega_{X_{0}}^{p}\right)
$$

the generalized semiregularity map.

The dimension of the Douady space $H_{X / \Sigma}$ at $[Z]$ satisfies

$$
\operatorname{dim}_{[Z]} H_{X / \Sigma} \geq \operatorname{dim}_{\mathbb{C}} T_{Z / X_{0}}^{1}\left(\mathcal{O}_{Z}\right)-\operatorname{dim}_{\mathbb{C}} \operatorname{ker} \sigma^{2}
$$

If $\sigma^{2}$ is injective, then $H_{X / \Sigma}$ is smooth over a closed subspace of $\Sigma$ in a neighbourhood of $[Z]$.

\section{The Singular Case}

In this announcement of results we restricted ourselves to smooth spaces $X$, but all the necessary ingredients

- Description of the obstruction for embedded deformations or for deformations of complexes of modules in terms of the Atiyah class,

- Construction of a trace for perfect complexes,

- Construction of the Atiyah-Chern character for perfect complexes,

have been established by L. Illusie in arbitrary generality in the algebraic case, and the analytic case can be handled through the methods of [Fle, OTT].

Using this machinery, one constructs first the cotangent complex $\mathbb{L}_{X / \Sigma}$ for an arbitrary morphism $X \rightarrow \Sigma$ of analytic spaces, schemes or even ringed topoi, secondly one defines traces $\operatorname{Ext}^{q}\left(\mathcal{F}, \mathcal{F} \otimes^{\mathbb{L}} \Lambda^{p} \mathbb{L}_{X / \Sigma}\right) \rightarrow \prod_{p} \mathrm{H}^{p+q}\left(X, \Lambda^{p} \mathbb{L}_{X / \Sigma}\right)$ for a perfect complex $\mathcal{F}$, which needs special care as $\Lambda^{p} \mathbb{L}_{X / \Sigma}$, the derived exterior powers of the cotangent complex, will in general not be perfect, and finally one defines the Atiyah class and, in characteristic zero, an Atiyah-Chern-Illusie character in $\prod_{p} \operatorname{Ext}^{p}\left(\mathcal{F}, \mathcal{F} \otimes^{\mathbb{L}} \Lambda^{p} \mathbb{L}_{X / \Sigma}\right)$ to obtain a commutative diagram extending 4.4) to something resembling a metatheorem:

Theorem 7.1. Let $X \rightarrow \Sigma$ be a morphism of complex spaces or of schemes over a field of characteristic zero. If $\mathcal{F}$ is a perfect complex on $X$ and $\mathcal{N}$ is a coherent 
$\mathcal{O}_{X}$-module then there is a commutative diagram

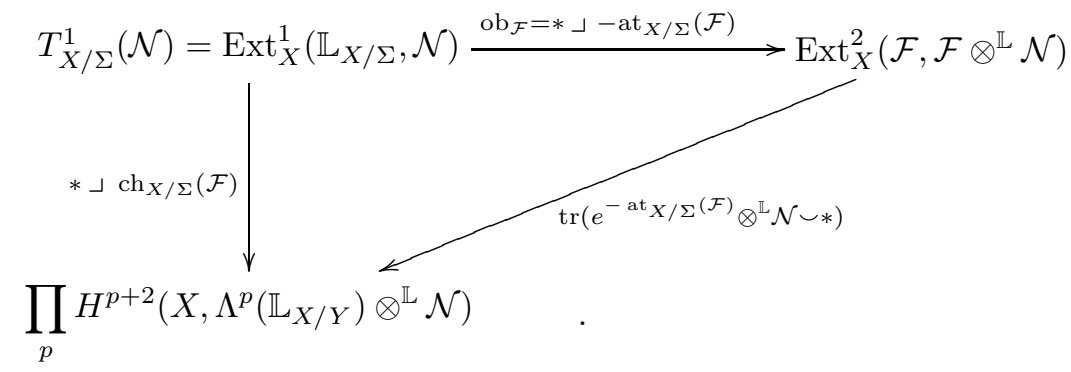

Moreover, if $\mathcal{F}=\mathcal{O}_{Z}$ for $Z \subseteq X$ a closed subspace, then the morphism on top, that is, contracting against the opposite of the Atiyah class, factors naturally through $T_{Z / X}^{2}\left(\left.\mathcal{N}\right|_{Z}\right)=\operatorname{Ext}_{Z}^{2}\left(\mathbb{L}_{Z / X}, \mathcal{O}_{Z} \otimes^{\mathbb{L}} \mathcal{N}\right)$.

\section{REFERENCES}

[ALG] Bourbaki, N.: Éléments de mathématique. Algèbre. Chapitres 1 à 3 . Hermann, Paris 1970

[ALJ] Angéniol, B.; Lejeune-Jalabert, M.: Calcul différentiel et classes caractéristiques en géométrie algébrique. Travaux en Cours 38, Hermann, Paris, 1989.

[Art] Artamkin, I. V.: On the deformation of sheaves. (Russian) Izv. Akad. Nauk SSSR Ser. Mat. 52 (1988), no. 3, 660-665, 672; translation in Math. USSR-Izv. 32 (1989), no. 3, 663-668.

[At] Atiyah, M. F.: Complex analytic connections in fibre bundles. Trans. Amer. Math. Soc. 85 (1957), 181-207.

[Blo] Bloch, S.: Semi-Regularity and de Rham Cohomology. Invent. Math. 17 (1972), 51-66.

[BFl] Buchweitz, R.-O.; Flenner, H.: A semiregularity map for modules and applications to deformations. preprint

[Del] Deligne, P.: Théorème de Lefschetz et critères de dégénérescence de suites spectrales. Inst. Hautes Études Sci. Publ. Math.35 (1968), 107-126.

[FGA] Grothendieck, A.: Fondements de la géométrie algébrique. [Extraits du Séminaire Bourbaki, 1957-1962.] Secrétariat mathématique, Paris 1962, ii+205 pp.

[FMa] Fantechi, B.; Manetti, M.: On the $T^{1}$-lifting theorem. J. Algebraic Geom. 8 (1999), no. 1, 31-39.

[Fle] Flenner, H.: Deformationen holomorpher Abbildungen. Habilitationsschrift, Osnabrück 1978

[Gre] Green, M. L.: Infinitesimal methods in Hodge theory. In: "Algebraic Cycles and Hodge Theory" (Torino, 1993), ed. by A. Albano, F. Bardelli, pp.1-92, Lecture Notes in Math., 1594, Springer-Verlag, Berlin-New York, 1994.

[Gr1] Grothendieck, A.: La théorie des classes de Chern. Bull. Soc. Math. France 86 (1958), $137-154$.

[Gr2] Grothendieck, A.: On the de Rham cohomology of algebraic varieties. Inst. Hautes Études Sci. Publ. Math.29 (1966), 95-103.

[Ill] Illusie, L.: Complexe cotangent et déformations I. Lecture Notes in Math. 239. Springer Verlag, Berlin-Heidelberg-New York 1971.

[Kaw1] Kawamata, Y.: Unobstructed deformations. A remark on a paper of Z. Ran: "Deformations of manifolds with torsion or negative canonical bundle" [J. Algebraic Geom. 1 (1992), no. 2, 279-291]; J. Algebraic Geom. 1 (1992), 183-190.

[Kaw2] Kawamata, Y.: Unobstructed deformations. II. J. Algebraic Geom. 4 (1995), 277-279.

[Kaw3] Kawamata, Y.: Erratum on: "Unobstructed deformations. A remark on a paper of Z. Ran: 'Deformations of manifolds with torsion or negative canonical bundle", [J. Algebraic Geom. 1 (1992), no. 2, 183-190]; J. Algebraic Geom. 6 (1997), 803-804.

[KSp] Kodaira, K.; Spencer, D. C.: A theorem of completeness of characteristic systems of complete continuous systems. Amer. J. Math. 81 (1959), 477-500.

[Muk] Mukai, S.: Symplectic structure of the moduli space of sheaves on an abelian or K3 surface. Invent. Math. 77 (1984), 101-116. 
[Mur] Murre, J. P.: Algebraic cycles and algebraic aspects of cohomology and K-theory. In: "Algebraic Cycles and Hodge Theory" (Torino, 1993), ed. by A. Albano, F. Bardelli, pp.93-152, Lecture Notes in Math., 1594, Springer-Verlag, Berlin-New York, 1994.

[OTT] O'Brian, N. R.; Toledo, D.; Tong, Y. L. L.: The trace map and characteristic classes for coherent sheaves. Amer. J. Math. 103 (1981), no. 2, 225-252.

[Pal] Palamodov, V. P.: Deformations of complex spaces. In: Several complex variables. IV. Algebraic aspects of complex analysis. Encyclopaedia of Mathematical Sciences 10, pp.105194. Springer-Verlag, Berlin-New York, 1990.

[Qui] Quillen, D.: On the (co-)homology of rings. Proc. Symp. in Pure Math., vol. XVII, pp. 65-87, Amer. Math. Soc. Providence, R.I., 1970.

[Ran1] Ran, Z.: Hodge theory and deformations of maps. Compositio Math. 97 (1995), no. 3, 309-328.

[Ran2] Ran, Z.: Hodge theory and the Hilbert scheme. J. Differential Geom. 37 (1993), no. 1, 191-198.

[Ran3] Ran, Z.: Deformations of manifolds with torsion or negative canonical bundle., J. Algebraic Geom. 1 (1992), 183-190.

[Ran4] Ran, Z.: Semiregularity, obstructions and deformations of Hodge classes. Preprint 1998

[Rim] Rim, D.S.: Formal deformation theory. In: Séminaire de Géometrie Algébrique, SGA 7. Lecture Notes in Mathematics 288, Springer Verlag Berlin-Heidelberg-New York 1972.

[Sev] Severi, F.: Sul teorema fondamentale dei sistemi continui di curve sopra una superficie algebrica. Ann. Mat. Pura Appl. 23 (1944), no. 4, 149-181.

[SGA 6] Théorie des intersections et théorème de Riemann-Roch. Séminaire de Géométrie Algébrique du Bois-Marie 1966-1967 (SGA 6). Dirigé par P. Berthelot, A. Grothendieck et L. Illusie. Avec la collaboration de D. Ferrand, J. P. Jouanolou, O. Jussila, S. Kleiman, M. Raynaud et J. P. Serre. Lecture Notes in Mathematics 225. Springer-Verlag, Berlin-New York, 1971.

[Ste] Steenbrink, J. H. M.: Some Remarks about the Hodge Conjecture. .Hodge theory (Sant Cugat, 1985), 165-175, Lecture Notes in Math. 1246, Springer-Verlag, Berlin-New York, 1987.

[Voi] Voisin, C.: Transcendental methods in the study of algebraic cycles. In: "Algebraic Cycles and Hodge Theory" (Torino, 1993), ed. by A. Albano, F. Bardelli, pp.153-222, Lecture Notes in Math., 1594, Springer, Berlin, 1994.

Dept. of Mathematics, University of Toronto, Toronto, Ont. M5S 3G3, Canada

E-mail address: ragnar@math.utoronto.ca

Fakultät für Mathematik, Ruhr-Universität Bochum, Geb. NA 2/72, 44781 Bochum, Germany

E-mail address: Hubert.Flenner@ruhr-uni-bochum.de 